\title{
The effect of conservation on the dynamics of chemical reaction networks
}

\author{
Katalin M. Hangos * Gábor Szederkényi *,** \\ * Process Control Research Group, Systems and Control Laboratory, \\ Computer and Automation Research Institute, \\ Hungarian Academy of Sciences \\ P.O. Box 63, H-1518 Budapest, Hungary \\ e-mail:\{hangos,szeder\}@scl.sztaki.hu \\ ** PPKE-ITK, 1083 Budapest, Práter u. 50/a, Hungary (e-mail: \\ szederkenyi@itk.ppke.hu)
}

\begin{abstract}
Component mass conservation gives a linear first integral to the dynamic reaction kinetic equations and greatly influences the set of admissible reactions. Therefore, mass conservation can be related to the deficiency of the reaction network and thus to its dynamic properties. In particular, it is shown that a mass conserving CRN without isomers and with a full rank complex composition matrix has zero deficiency. The concepts and results are illustrated on the example of the original and the lumped version of Michaelis-Menten kinetics.
\end{abstract}

Keywords: Process control; Chemical variables control; Modelling; Graph theoretic models; Structural stability;

\section{INTRODUCTION}

It is well-known that kinetic systems form a wide class of nolinear models with good dynamical descriptive properties [3]. Therefore (bio)chemical reaction networks (CRNs) are often used to model complex biological mechanisms [11], or even to describe dynamical systems in application fields seemingly far from chemistry such as mechanical or electrical systems [12]. The increasing and extended interest towards this field is shown by the fact that numerous surveys and tutorials have been published even in journals where the primary scope is not chemistry $[1,2,13]$.

The classical chemical theory of deterministic CRNs obeying the mass action law (MAL CRNs) assumes closed isotherm and isobaric conditions, where the total mass is conserved. This mass conservation applies also for the set of species that participate in any chemical reaction. It is intuitively clear, that a MAL CRN system with these mass conservations should not produce any "exotic" nonlinear behavior, but will be globally stable within each stoichiometric compatibility class.

The mathematical generalization of MAL CRN systems, however, does not require conservation assumptions, that allows the description of various nonlinear behaviour like multiplicities, limit cycles or even chaotic phenomena. Naturally, this generalization makes the MAL CRN system class to be a wide class of smooth positive nonlinear systems.

\footnotetext{
^ This work was supported in part by the Hungarian Research Fund through grant 83440 , and by the by the Control Engineering Research Group of the Budapest University of Technology and Economics.
}

The aim of this paper is to investigate the dynamic consequences of conservations on the qualitative properties of MAL CRN dynamics.

\section{BASIC NOTIONS}

Consider a CRN that obeys the mass action law. The structure of the CRN is uniquely given in terms of its Kirchhoff matrix $A_{k}$ and its complex composition matrix $Y \in \mathbb{Z}_{+0}^{n \times m}$ with non-negative integer elements, the columns of which describe the composition of its complexes $C_{i}, i=1, \ldots, m$ over its species $X_{j}, j=1, \ldots, n$. The Kirchhoff matrix $A_{k} \in \mathbb{R}^{m \times m}$ describes the structure of chemical reactions $C_{i} \mapsto C_{j}$ with the reaction rate coefficient $k_{i j}>0$.

The following general assumptions are considered throughout this paper.

(G1) There is no isolated (i.e. unconnected) complex in the CRN.

(G2) There is no inert (i.e. non-reacting) specie in the system.

(G3) $Y$ is of full rank.

Assumption (G3) implies that

- the columns of $Y$ are linearly independent in the $n \geq m$ case

- the rows of $Y$ are linearly independent in the $n \leq m$ case

Both the reaction graph determined by the matrix $A_{k}$ and the properties of $Y$ influence the structural dynamical properties of a CRN. Let us introduce the following notations. 


$$
[Y]_{i j}=\alpha_{i j} \quad, \quad\left[A_{k}\right]_{i j}=\left\{\begin{array}{cl}
-\sum_{l=1}^{m} k_{i l} & \text { if } i=j \\
k_{j i} & \text { if } i \neq j
\end{array}\right.
$$

With the above matrices the time evolution of the specie concentrations $x=\left[x_{1}, \ldots, x_{n}\right]^{T}$ is described by the following set of ODEs:

$$
\frac{d x}{d t}=Y A_{k} \varphi(x) \quad, \quad \varphi_{i}(x)=\prod_{j=1}^{m} x_{j}^{\alpha_{i j}}
$$

\subsection{The reaction graph}

The vertexes $V$ of the reaction graph $G=(V, E)$ correspond to the complexes, and the edges $E$ to the reactions. Two complexes $C_{k}$ and $C_{l}$ are connected by a directed edge $C_{k} C_{l}$, if a reaction in the form of $C_{k} \mapsto C_{l}$ exists. Edge weights can be associated to the edges that are the reaction rate constants $k_{k l}>0$, thus he reaction graph is a weighted directed graph.

Note that the Kirchhoff matrix $A_{k}$ of a CRN uniquely determines its reaction graph and vice versa. However, the Kirchhoff matrix of the reaction graph does not uniquely determine the reaction kinetic system itself, since the information on the composition of the complexes is missing from the graph: it is contained in the complex composition matrix $Y$.

\subsection{The deficiency of a $C R N$}

The stoichiometric subspace First we define the set of reaction vectors as: $\mathcal{R}=\left\{\rho^{(l, k)}=\eta^{(l)}-\eta^{(k)} \mid C_{k} C_{l} \in\right.$ $E$ in $G$, where $\eta^{(i)}$ denotes the $i$ th column of $Y$.

The deficiency is an integer number which depends on the properties of matrix $Y$, and on the structure of the reaction graph $G$. The deficiency $\delta$ is defined as:

$$
\delta=m-\ell-s
$$

where $m$ is the number of complexes and $\ell$ is the number of connected components in the reaction graph, while $s$ is the dimension of the stoichiometric sub-space, i.e. $s=\operatorname{rank}(\mathcal{R})$.

The deficiency zero theorem An important structural property of CRNs is (weak) reversibility. A CRN is called weakly reversible if whenever there exists a directed path from complex $C_{i}$ to $C_{j}$ in the reaction graph, then there exists a directed path from $C_{j}$ to $C_{i}$. The main result of the well-known deficiency zero theorem is the parameterindependent robust stability of weakly reversible deficiency zero CRNs. The exact form of the theorem is taken from [6].

Deficiency Zero Theorem. For any reaction network of deficiency zero the following statements hold true:

(1) If the network is not weakly reversible then, for arbitrary kinetics (not necessarily mass action), the differential equations for the corresponding reaction system cannot admit a positive steady state (i.e. a steady state in $\left.\mathbb{R}_{+}^{n}\right)$.
(2) If the network is not weakly reversible then, for arbitrary kinetics (not necessarily mass action), the differential equations of the corresponding reaction system cannot admit a cyclic composition trajectory along which all species concentrations are positive.

(3) If the network is weakly reversible then, for mass action kinetics (but regardless of the positive values the reaction rate coefficients take), the differential equations of the corresponding reaction system have the following properties: There exists within each positive stoichiometric compatibility class precisely one steady state; that steady state is asymptotically stable; and there is no nontrivial cyclic composition trajectory along which all species concentrations are positive.

\subsection{Conservation equations}

Mass conservative chemical reactions Let us assume that the given set of chemical reactions is physically plausible, i.e. each reaction obeys the mass conservation. This means, that for each reaction $C_{l} \mapsto C_{p}$, the following equality holds

$$
\sum_{i=1}^{n} \alpha_{i l} \mathcal{M}_{i}=\sum_{i=1}^{n} \alpha_{i p} \mathcal{M}_{i}=c_{s},
$$

where $\mathcal{M}_{i}>0$ is the molecular weight of the component $X_{i}, l$ and $p$ are column indexes in $Y$ and $c_{s}>0$ is a constant weighted column-sum. Let us form a row vector $M \in \mathbb{R}^{n}$ from the molecular weights, then the above equation can be written as

$$
M \cdot \eta^{(l)}=M \cdot \eta^{(p)}=c_{s}, \text { or } M \cdot \rho^{(l, p)}=0
$$

where $\rho^{(l, p)}$ is the corresponding reaction vector. A set of reactions with the above property will be called mass conservative reaction set.

The zero complex is a special complex that is used to describe the environment with infinite source and sink capacity for all components. The zero complex $\emptyset$ is described algebraically by a zero column vector in $Y$, i.e. $\eta^{(0)}=\underline{0}_{n}^{T} \in \mathbb{R}^{n}$. Eq. (4) shows that a reaction in the form of $C_{i} \mapsto \emptyset$ or $\emptyset \mapsto C_{i}$ cannot be part of a mass conservative reaction set.

Conservation in the specie concentration space The column conservation property of $A_{k}$ can be expressed algebraically as $\underline{1}_{m} \cdot A_{k}=\underline{0}_{m}$, where $\underline{1}_{m}$ is the $m$-elements row vector with identically 1 entries. In addition, equation (4) states that the weighted column-sum of any column in $Y$ is the same $\left(c_{s}\right)$, where the weights are the molecular weights $\mathcal{M}_{i}$. Therefore, we can form a weighted columnsummation vector $w$ such that

$$
w \cdot Y=\underline{1}_{m} \Rightarrow w \cdot Y \cdot A_{k}=\underline{0}_{m}
$$

with $w=\left[\frac{\mathcal{M}_{1}}{c_{s}}, \ldots, \frac{\mathcal{M}_{n}}{c_{s}}\right]$, that is the weighted mass conservation property in the specie concentration space.

\section{THE EFFECT OF THE CONSERVATION EQUATIONS ON THE STRUCTURAL PROPERTIES OF A CRN}

The conservation equations (4) or (5) show that they put an algebraic constraint on the columns of the complex 
composition matrix $Y$. At the same time, they partition the set of reactions that is reflected in the linkage classes of the CRN. Therefore, two different cases will be considered: first reaction graphs forming a single linkage class (i.e. connected component) is investigated, thereafter the multiple connected component case is discussed. The effect of conservation equations on the deficiency of the CRN will be investigated, because it may have a determining effect on its dynamic properties.

\subsection{The mass conservation relation between complexes}

Based on the above, we say that two complexes $C_{i}$ and $C_{j}$ in a CRN are in a mass conserving relation if there exists an (elementwise) strictly positive vector $M$ such that $M \eta^{(i)}=M \eta^{(j)}$. Clearly, any reaction $C_{i} \rightarrow C_{j}$ obeys the mass conservation if and only if $C_{i}$ and $C_{j}$ are in a mass conserving relation, and this property only depends on the $i$ th and $j$ th columns of the complex composition matrix $Y$. Naturally, it is not enough for a CRN to be mass-conserving that the reacting complexpairs are individually in a mass conserving relation. E.g. the following simple CRN consists of reacting complexpairs in mass conserving relations, although the entire network is not mass conserving:

$$
\begin{aligned}
X_{1}+X_{2} & \rightarrow X_{3} \\
X_{3} & \rightarrow 2 X_{4} \\
X_{1}+X_{2} & \rightarrow X_{4} .
\end{aligned}
$$

Therefore, it is possible only in certain cases to decide whether a CRN violates mass conservation by inspecting the reactions individually (see the examples later).

With a fixed $M$, the mass conserving relation is clearly symmetric and transitive (and it is also reflexive, if we consider complexes trivially reacting to themselves). Obviously, an entire CRN is mass-conservative if and only if all reacting complex pairs are in mass conserving relations with one common positive $M$.

Note that our chemically motivated notion of mass conservation is not equivalent to the definition that is often used in chemical reaction network theory. According to the latter, a CRN is sometimes called mass-conservative if and only if there exists a strictly positive vector in the kernel of $\left(Y \cdot A_{k}\right)^{T}[7]$. Consider the simple network in Eq. (7) where all the rate coefficients are 1 for simplicity. Then, the $Y$ and $A_{k}$ matrices of the network are the following:

$$
Y=\left[\begin{array}{llll}
1 & 0 & 0 & 0 \\
1 & 0 & 0 & 0 \\
0 & 1 & 0 & 0 \\
0 & 0 & 2 & 1
\end{array}\right], \quad A_{k}=\left[\begin{array}{cccc}
-2 & 0 & 0 & 0 \\
1 & -1 & 0 & 0 \\
0 & 1 & 0 & 0 \\
1 & 0 & 0 & 0
\end{array}\right]
$$

It can be checked that the positive vector

$$
\left[\begin{array}{llll}
0.7693 & 0.0265 & 1.0611 & 0.5306
\end{array}\right]^{T}
$$

is in the kernel of $\left(Y \cdot A_{k}\right)^{T}$ but the CRN is not mass conserving according to our definition.

\subsection{The single connected component case}

Assume we have a CRN with a mass conservative reaction set (implying the absence of the zero complex). Recalling that isolated complexes are not allowed, with $m$ complexes we should have at least $r_{m}=m-1$ reactions in the single connected component (i.e. $\ell=1$ ) case. Moreover, the constant $c_{s}>0$ in Eq. (5) is the same for all reactions, and the Kirchhoff matrix $A_{k}$ consists of a single columnconservation block in this case.

$A$ simple example A simple reaction graph with $n=3$ species $(A, B, C)$ and $m=3$ complexes is shown in Fig. 1. The minimum set of reactions with reaction vectors

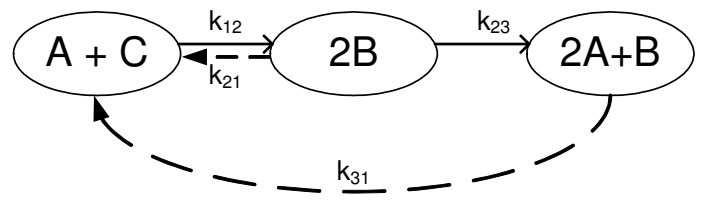

Fig. 1. The structure graph of the simple example of a single linkage class

$$
\rho^{(1,2)}=\left[\begin{array}{c}
1 \\
-2 \\
1
\end{array}\right] \quad, \quad \rho^{(2,3)}=\left[\begin{array}{c}
-2 \\
1 \\
0
\end{array}\right]
$$

is denoted by continuous edge lines in the figure.

The maximum number of complexes in a single connected component with a mass conservative reaction set can be determined by the defining equation (5) $M \cdot \eta^{(j)}=c_{s}, j=$ $1, \ldots, m$ where $\eta^{(j)} \in \mathbb{Z}_{+0}^{n}$ is the column of the matrix $Y$ corresponding to the complex $C_{j}$, and $M^{T} \in \mathbb{R}^{n}$ is the vector of the molecular weights with positive elements. There are two possible cases to consider.

(i) $m \leq n$

As $Y$ is of full rank, the complexes form a linearly independent set of column vectors, and there is infinitely many such molecular weight vectors $M$ that satisfies Eq.(5). (In the case of $m=n$ and fixed $c_{s}$ one has a unique solution $M$ ).

(ii) $m>n$

Then, with $c_{s}$ and $M$ fixed, we can have maximum $n$ different and linearly independent complexes, since the linear set of equations

$$
M \cdot Y^{*}=\left[c_{s}, c_{s}, \ldots, c_{s}\right]
$$

would uniquely determine $M$ from a given $Y^{*} \in$ $\mathbb{Z}_{+}^{n \times n}$, where we have collected the set of linearly independent complex vectors to the matrix $Y^{*}$.

We shall now show that we can not have more complexes corresponding to a column of $\eta(j), j>n$ from the rest of $Y$ that is both linearly dependent of the columns of $Y^{*}\left(\eta^{(1)}, \ldots, \eta^{(n)}\right)$ and satisfies $M$. $\eta^{(j)}=c_{s}$. Let us assume that this dependent column of $Y$ is in the form of a linear combination

$$
\eta^{(j)}=\varphi_{1} \eta^{(1)}+\ldots+\varphi_{n} \eta^{(n)}
$$

If we multiply both sides by the row vector $M$, the equation

$$
c_{s}=c_{s}\left(\varphi_{1}+\ldots+\varphi_{n}\right) \Longrightarrow \varphi_{1}+\ldots+\varphi_{n}=1
$$

is obtained. But the coefficients in the linear combination (11) are uniquely determined, therefore they can satisfy the additional constraint (12) originating form the mass conservation only in a very special fortunate case when $\mathcal{M}_{i}=\mathcal{M}_{k}$ for some $(i, k)$ specie 
pair. The corresponding species $X_{i}$ and $X_{k}$ are then called isomers in the chemical terminology.

An example of isomers Let us have only two species $X_{1}$ and $X_{2}$ in a $\mathrm{CRN}$, and let $\mathcal{M}_{1}=\mathcal{M}_{2}=1$ with $c_{s}=2$. Then the columns of the complex composition matrix with three complexes $2 X_{1}, 2 X_{2}, X_{1}+X_{2}$

$$
Y=\left[\begin{array}{lll}
2 & 0 & 1 \\
0 & 2 & 1
\end{array}\right]
$$

satisfy both Eq. (11) and the additional constraint (12) when $3=m>n=2$.

Deficiency Now we only need to consider the $n \geq m$ case. Because of the full rank property of $Y$ the columns of it are linearly independent and span a maximum $m$ dimensional stoichiometric subspace, i.e. $s=\operatorname{rank}(\mathcal{R}) \leq m$. At the same time we have $s \geq m-1$ because of the minimum number of reactions.

If $r \geq m$ then the conservation equations (5) put an additional linear relationship to the reaction vectors, therefore $s=m-1$, otherwise $s=r_{m}=m-1$. Therefore, in both cases we have $\delta=m-\ell-s=m-1-m+1=0$.

Simple example continued Fig. 1 shows that $m=n=3$ in this case. If one chooses the molecular weight of specie $A$ to be 1 , then the molecular weight vector is $M=\left[\begin{array}{lll}1 & 2 & 3\end{array}\right]$, and $c_{s}=4$. The reaction vectors in Eq. (9) span the stoichiometric sub-space, therefore $s=2$ and $\delta=0$.

Is is easy to see that the dimension of the stoichiometric sub-space will not increase if one adds any further reaction to the CRN. For example, the reaction vectors of two reactions denoted by dashed lines in Fig. 1 can be expressed as $\rho^{(2,1)}=-\rho^{(1,2)}$, and $\rho^{(3,1)}=-\left(\rho^{(1,2)}+\rho^{(2,3)}\right)$.

\subsection{Multiple connected component cases}

Assume a mass action law CRN with only mass conservative reactions that has $\ell$ linkage classes, i.e. $\ell$ connected components in its reaction graph. Then the set of complexes are partitioned according to these linkage classes, where the partitions have $m_{j}, j=1, \ldots, \ell$ members, respectively, where $m=m_{1}+\ldots+m_{\ell}$.

Determination of the molecular weights As chemical reactions connect only complexes within the same linkage class $L_{j}$, the conservation equation $M \cdot \eta^{(i)}=c_{s}^{(j)}$ is valid only if the corresponding complex $C_{i}$ is in $L_{j}$, and the constant $c_{s}^{(j)}$ may be different for different linkage classes. However, for any reaction vector $\rho^{(i, p)}$ the conservation equation has the same form $M \cdot \rho^{(i, p)}=0$. This enables to compute the molecular weight vector $M$ that is joint for the entire CRN as follows.

Let us form the reaction matrix $R$ from the reaction vectors $\rho^{(i, p)}, C_{i} C_{p} \in E$ of the reaction graph $G$. The rank of this matrix is equal to the dimension of the stoichiometric sub-space, i.e. $n \geq s=\operatorname{rank}(R)$. At the same time, the reaction vectors are formed as differences of the component vectors $\rho^{(i, p)}=\eta^{(i)}-\eta^{(p)}$, thus the rank of $R$ is is constrained by $n \geq \operatorname{rank}(Y) \geq \operatorname{rank}(R)$. Now we can form the matrix $R^{*}$ from a set of $s$ linearly independent reaction vectors, that gives rise to the linkage class independent form of the conservation equations

$$
M \cdot R^{*}=[0,0, \ldots 0]
$$

The elements $\mathcal{M}_{i}>0$ of the molecular weight vector can be determined from the above equation by fixing $n-s-1$ molecular weights $\mathcal{M}_{i}$ (such that $\mathcal{M}_{i} \neq \mathcal{M}_{p}$ for $i \neq p$ ) that we could uniquely determine the rest from Eq. (13).

Then for each linkage class $L_{j}$ we can compute the constant $c_{s}^{(j)}$ by finding a complex $\eta^{(i)}$ from this class and computing $M \cdot \eta^{(i)}=c_{s}^{(j)}$.

The maximal dimension of the stoichiometric sub-space Because of the above linkage class independent form of the conservation equations (13), and the given constant positive nature of the component weight vector $M \in \mathbb{R}_{+}^{n}$, the maximal number of the linearly independent reaction vectors is $n-1$. This implies that the maximal dimension of the stoichiometric sub-space $s_{m}=n-1$.

The maximal set of the linearly independent reaction vectors can also be partitioned according to the linkage class they belong to, as no reaction is taking place between complexes in different linkage classes. Let the number of such reactions belonging to the linkage class $L_{j}$ be denoted by $r_{j} \geq 1$, such that $r_{1}+\ldots+r_{\ell}=s \leq n-1$. Here we have accounted for the fact that the stoichiometric subspace may not be maximal.

The number of complexes in a linkage class induced by the set of linearly independent reaction vectors can be determined from the fact, that at least $m_{j}=r_{j}+1$ linearly independent column vectors $\eta^{i}$ corresponding to complexes $C_{i}$ are needed to generate this set and to form a connected component with $r_{j}$ edges. On the other hand, if we added new different complexes within this linkage class then the number of linearly independent reaction vectors would increase, therefore we could only add new reactions between the existing complexes to keep this situation. Finally we conclude that $r_{j}$ linearly independent reaction vector induces $m_{j}=r_{j}+1$ linearly independent complexes.

The overall number of complexes can be computed as

$$
m=\sum_{j=1}^{\ell} m_{j}=\sum_{j=1}^{\ell}\left(r_{j}+1\right)=s+\ell
$$

The deficiency Now we can assemble the above results to determine the deficiency of the CRN with only mass conservative reactions that has $\ell$ linkage classes. This is computed by the definition as $\delta=m-s-\ell=s+\ell-s-$ $\ell=0$ using Eq. (14). 


\section{CASE STUDY: CONSERVATION IN THE VERSIONS OF THE MICHAELIS-MENTEN REACTION SCHEME}

The aim of this section is to show how the model reduction (or simplification) transformation can destroy the conservation properties of an originally physically plausible model, and what are the structural and dynamic consequences of this. The detailed description of the MichaelisMenten reaction scheme and the applied model reduction transformations can be found in [8].

\subsection{The original full scheme}

The Michaelis-Menten reaction scheme describes a simple enzyme-kinetic reaction with the following reaction and dynamic model equations

$$
\begin{aligned}
E+S & \rightleftarrows E S \quad E S \rightleftarrows E+P \quad E S+S \rightleftarrows E S S \\
E: & \frac{d x_{1}}{d t}=-k_{1}^{+} x_{1} x_{2}+k_{1}^{-} x_{3}-k_{2}^{-} x_{1} x_{5}+k_{2}^{+} x_{3} \\
S: & \frac{d x_{2}}{d t}=-k_{1}^{+} x_{1} x_{2}+k_{1}^{-} x_{3}-k_{3}^{+} x_{2} x_{3}+k_{3}^{-} x_{4} \\
E S: & \frac{d x_{3}}{d t}=+k_{1}^{+} x_{1} x_{2}-k_{1}^{-} x_{3}+k_{2}^{-} x_{1} x_{5}-k_{2}^{+} x_{3}- \\
& -k_{3}^{+} x_{2} x_{3}+k_{3}^{-} x_{4} \\
E S S: & \frac{d x_{4}}{d t}=+k_{3}^{+} x_{2} x_{3}-k_{3}^{-} x_{4} \\
P: & : \frac{d x_{5}}{d t}=-k_{2}^{-} x_{1} x_{5}+k_{2}^{+} x_{3}
\end{aligned}
$$

where the concentration state vector $x$ is formed as

$$
x_{1}=[E], x_{2}=[S], x_{3}=[E S], x_{4}=[E S S], x_{5}=[P]
$$

from the species $E$ for the enzyme, $S$ for the substrate, $P$ for the product, and $E S$ and $E S S$ being intermediate chemical complexes.

The dynamical behavior of the concentration variables and the reaction graph that consists of two reversible connected components are seen in figure 2 , with the following model parameters used for the simulations:

$k_{1}^{+}=1, k_{1}^{-}=0.8, k_{2}^{+}=1.1, k_{2}^{-}=0.75, k_{3}^{+}=2, k_{3}^{-}=1.8$

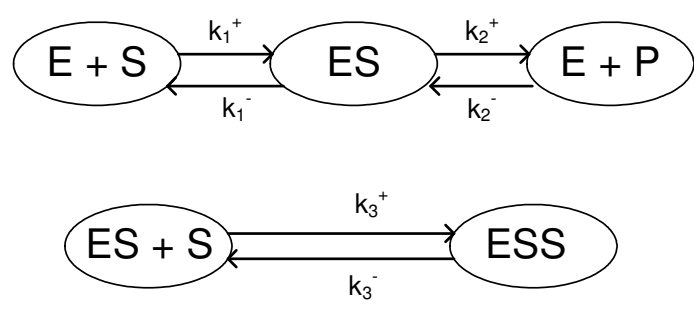

Fig. 2. The structure graph of the Michaelis-Menten reaction scheme

Conservation analysis This CRN clearly obeys the mass conservation. Now we have two "free" molecular weights that we can fix, that are $M_{1}=\mathcal{M}_{E}=2$ and $M_{2}=\mathcal{M}_{S}=$ 1. Then a vector $M$ obeying the mass conservation is

$$
M=\left[\begin{array}{lllll}
2 & 1 & 3 & 4 & 1
\end{array}\right]
$$

and the constants are $c_{s}^{(1)}=3$ and $c_{s}^{(2)}=4$.
The deficiency is computed from the definition, with $m=5, \ell=2$ and $s=3$. Then the deficiency of this reversible model is zero, therefore its equilibrium points are stable because of the deficiency zero theorem.

\subsection{The lumped reaction scheme}

The two intermediate species $E S$ and $E S S$ with similar dynamics are lumped to form the lumped pseudocomponent $E_{S}$ with concentration $\bar{x}_{3}=\left[E_{S}\right]$. Then the equations needed for the transformation are

$$
\bar{x}_{3}=x_{3}+x_{4} \quad, \quad x_{3}=\frac{1}{2} \bar{x}_{3} \quad, \quad x_{4}=\frac{1}{2} \bar{x}_{3}
$$

and the concentration variable $x_{4}$ is left out from the model. The idea behind this transformation is that specie concentrations with "similar" dynamics are essentially approximately identical, that is seen from (15).

The reduced kinetic equations are

$$
\begin{aligned}
& \frac{d x_{1}}{d t}=-k_{1}^{+} x_{1} x_{2}+\frac{1}{2} k_{1}^{-} \bar{x}_{3}+\frac{1}{2} k_{2}^{+} \bar{x}_{3}-k_{2}^{-} x_{1} x_{5} \\
& \frac{d x_{2}}{d t}=-k_{1}^{+} x_{1} x_{2}+\frac{1}{2} k_{1}^{-} \bar{x}_{3}+\frac{1}{2} k_{3}^{-} \bar{x}_{3}-\frac{1}{2} k_{3}^{+} x_{2} \bar{x}_{3} \\
& \frac{d \bar{x}_{3}}{d t}=k_{1}^{+} x_{1} x_{2}-\frac{1}{2} k_{1}^{-} \bar{x}_{3}-\frac{1}{2} k_{2}^{+} \bar{x}_{3}+k_{2}^{-} x_{1} x_{5} \\
& \frac{d x_{5}}{d t}=\frac{1}{2} k_{2}^{+} \bar{x}_{3}-k_{2}^{-} x_{1} x_{5}
\end{aligned}
$$

It is important to note that the number of complexes has also been reduced by one, as both $E S$ and $E S S$ formed complexes that were replaced by a new complex formed by $E_{S}$. Thus the new complex composition matrix $Y$ is in the form

$$
Y=\left[\begin{array}{llll}
1 & 0 & 0 & 0 \\
1 & 0 & 0 & 1 \\
0 & 1 & 0 & 1 \\
0 & 0 & 1 & 0
\end{array}\right]
$$

and the reaction graph is depicted in Fig. 3

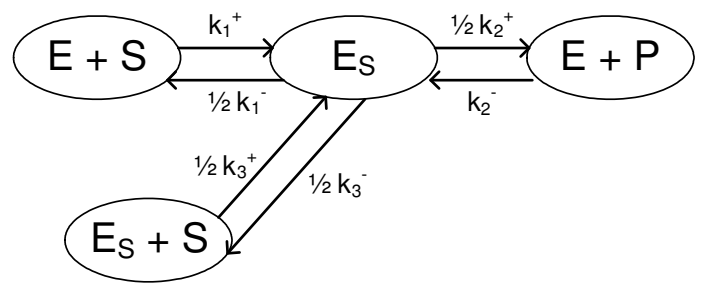

Fig. 3. Reaction graph of the lumped Michaelis-Menten scheme

Conservation analysis This CRN does not obey the mass conservation, that is seen from the presence of the reaction

$$
E_{S}+S \rightleftarrows E_{S}
$$

in the network. The reaction vector $\rho^{(*)}=\eta^{(4)}-\eta^{(2)}$ corresponding to this reaction pair is $\rho^{(*)}=\left[\begin{array}{llll}0 & 1 & 0 & 0\end{array}\right]^{T}$, that contains only a positive elements besides of the zero ones, and thus can not fulfil the conservation constraint $M \rho^{(*)}=0$ for any positive molecular weight vector $M$. 
Deficiency and structural stability Because of the condensation of two complexes, the number of complexes is now $\bar{m}=4$, and the number of linkage classes is also reduced by one, i.e. $\bar{\ell}=1$. The dimension of the stoichiometric space remains $s=3$, therefore the reduced model is of zero deficiency, and therefore it is globally stable (we remark that the modified CRN consists of only one linkage class).

\subsection{A simplified scheme by using quasi steady state for the} specie $E S$

Let us denote the steady state value of specie $E S$ by $x_{3}^{*}$, and let us notice that $E S$ forms a complex in itself. Therefore, the reduced complex composition matrix $\bar{Y}$ becomes

$$
\bar{Y}=\left[\begin{array}{lllll}
1 & 0 & 1 & 0 & 0 \\
1 & 0 & 0 & 1 & 0 \\
0 & 0 & 0 & 0 & 1 \\
0 & 0 & 1 & 0 & 0
\end{array}\right]
$$

that shows that the zero complex appears in the reaction kinetic system (see 2 nd column of $\bar{Y}$ ). The reduced kinetic equations are

$$
\begin{aligned}
& \frac{d x_{1}}{d t}=-k_{1}^{+} x_{1} x_{2}+\bar{k}_{1}^{-}-k_{2}^{-} x_{1} x_{5}+\bar{k}_{2}^{+} \\
& \frac{d x_{2}}{d t}=-k_{1}^{+} x_{1} x_{2}+\bar{k}_{1}^{-}-\bar{k}_{3}^{+} x_{2}+k_{3}^{-} x_{4} \\
& \frac{d x_{4}}{d t}=\bar{k}_{3}^{+} x_{2}-k_{3}^{-} x_{4} \\
& \frac{d x_{5}}{d t}=-k_{2}^{-} x_{1} x_{5}+\bar{k}_{2}^{+}
\end{aligned}
$$

where the new reaction rate constants are formed as $\bar{k}_{i}^{ \pm}=$ $k_{i}^{ \pm} x_{3}^{*}$.

The reaction graph of the reduced system is seen in Fig. 4 .

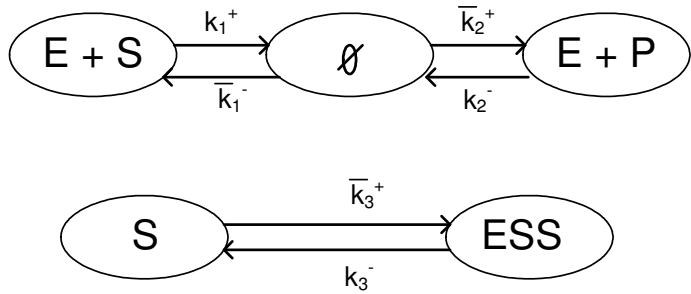

Fig. 4. Reaction graph of the reduced Michelis Menten kinetics with $E S$ in quasi steady state

Conservation analysis For the zero complex that appears in the place of $E S$ there is no component mass balance, but the presence of constant positive terms indicate that the systems becomes open.

\section{CONCLUSIONS AND FUTURE WORK}

In this paper it was shown, that a mass action law CRN with only mass conservative reactions, with a full-rank complex composition matrix $Y$ and without isomers is necessarily of zero deficiency. Therefore, a weakly reversible conservative CRN having the above mentioned properties possess a unique stable positive equilibrium in each stoichiometric compatibility class.

The mass conservation property can be checked by algebraic methods using only the molecular weights of the components and the complex composition matrix $Y$, but it has implications on the possible set of complexes and on the chemical reactions that may occur between them, i.e. on the Kirchhoff matrix $A_{k}$.

Further analysis is needed to find out how one can recognize the presence of non mass conservative reactions in the network (besides of detecting the zero complex), and - when this fact is not by the intention of modeler - how one can "repair" a non-conservative network when this property is not realistic and/or non-desired.

\section{REFERENCES}

[1] D. Angeli. A tutorial on chemical network dynamics. European Journal of Control, 15:398-406, 2009.

[2] V. Chellaboina, S. P. Bhat, W. M. Haddad, and D. S. Bernstein. Modeling and analysis of mass-action kinetics - nonnegativity, realizability, reducibility, and semistability. IEEE Control Systems Magazine, 29:6078, 2009.

[3] P. Érdi and J. Tóth. Mathematical Models of Chemical Reactions. Theory and Applications of Deterministic and Stochastic Models. Manchester University Press, Princeton University Press, 1989

[4] M. Feinberg. On chemical kinetics of a certain class. Arch. Rational Mech. Anal., 46:1-41, 1972.

[5] M. Feinberg. Lectures on chemical reaction networks. Notes of lectures given at the Mathematics Research Center, University of Wisconsin, 1979.

[6] M. Feinberg. Chemical reaction network structure and the stability of complex isothermal reactors I. The deficiency zero and deficiency one theorems. Chemical Engineering Science, 42:2229-2268, 1972.

[7] J. Gunawardena. Chemical Reaction Network Theory for In-silico Biologists. Bauer Center For Genomics Research, Harvard University, Cambridge, MA, 2003.

[8] K. M. Hangos Engineering Model Reduction and Entropy-based Lyapunov Functions in Chemical Reaction Kinetics. Entropy, 12:772-797, 2010.

[9] K. M.Hangos and I. T. Cameron. Process modelling and model analysis. Academic Press, 2001.

[10] K. M. Hangos and G. Szederkényi. The underlying linear dynamics of some positive polynomial systems. Physics Letters A, 376:3129-3134, 2012.

[11] G. Nicolis and I. Prigogine. Self-organization in Nonequilibrium Systems: From Dissipative Structures to Order through Fluctuations. John Wiley and Sons, New York, 1977.

[12] N. Samardzija, L. D. Greller, and E. Wassermann. Nonlinear chemical kinetic schemes derived from mechanical and electrical dynamical systems. Journal of Chemical Physics, 90 (4):2296-2304, 1989.

[13] E. Sontag. Structure and stability of certain chemical networks and applications to the kinetic proofreading model of T-cell receptor signal transduction. IEEE Trans. Autom. Control, 46:1028-1047, 2001. 